\title{
Use of water-miscible retinyl palmitate as markers of chylomicrons gives earlier peak response of plasma retinyl esters compared with oil-soluble retinyl palmitate
}

\author{
K. D. Renuka R. Silva, Christine M. Williams and Julie A. Lovegrove* \\ Hugh Sinclair Unit of Human Nutrition, School of Food Biosciences, University of Reading, Reading, Berkshire RG6 6AP, UK
}

(Received 12 January 2001 - Revised 1 June 2001 - Accepted 11 June 2001)

\begin{abstract}
Delayed peak response of plasma retinyl esters (RE) relative to plasma triacylglycerols (TAG) and apolipoprotein (Apo) B-48 responses following a fat load supplemented with vitamin A raised doubts about the use of vitamin A to label dietary-derived lipids and lipoproteins. The present study compared the use of water-miscible and oil-soluble retinyl palmitate (RP) as markers of dietary-derived lipoproteins in healthy subjects along with the measurements of postprandial plasma TAG and ApoB-48 responses to investigate whether the delayed peak response observed was due to delayed intestinal output of RE from oil-based solutions. Nine healthy female subjects were given a standard test meal containing a dose $(112 \mathrm{mg})$ of RP in either water-miscible or oil-soluble form in random order, on two separate occasions after a $12 \mathrm{~h}$ overnight fast. The results showed that the mean plasma RE concentrations reached a peak significantly later than mean plasma TAG and ApoB-48 concentrations when oil-soluble RP was consumed, whereas plasma RE peaked earlier relative to plasma TAG and ApoB-48 responses when water-miscible RP was used. The results suggested a more rapid absorption with a significantly higher and earlier peak response of plasma RE when water-miscible RP was consumed. This was in contrast to the delayed initial appearance and later sustained higher concentrations of plasma RE during the late postprandial period when oil-soluble RP was consumed. The RE response to the water-miscible RP showed better concordance with plasma TAG response than that of oil-soluble RP.
\end{abstract}

Retinyl esters: Apolipoprotein B-48: Triacylglycerol: Chylomicron

With greater understanding and recognition of the role of chylomicrons (CM) and their remnants in the pathogenesis of CHD (Zilversmit, 1979), the need for a specific method to distinguish intestinally-derived lipoproteins from the structurally similar hepatic VLDL has been emphasised.

Use of vitamin A has been shown by many investigators to be a convenient and established method to label intestinally-derived CM particles (Hazzard \& Bierman, 1976; Wilson et al. 1983; Cortner et al. 1987). When vitamin $\mathrm{A}$ is ingested in esterified form, it is hydrolysed in the small intestine to retinol and is taken up by enterocytes. Retinol is re-esterified and incorporated into the core of CM as retinyl esters (RE) along with triacylglycerols (TAG) (Goodman et al. 1965, 1966). RE are associated with CM and CM remnants until they are taken up by the liver by a receptor-mediated uptake and not re-secreted by the liver in free form (Thompson et al. 1983). The presence and concentration of $\mathrm{RE}$ in the plasma after vitamin A administration has been used as an indicator of CM and $\mathrm{CM}$ remnants. The other method of measuring $\mathrm{CM}$ and $\mathrm{CM}$ remnants is the determination of the characteristic structural protein of the intestinally-derived lipoproteins, apolipoprotein (Apo) B-48, which is widely accepted as the most appropriate method of quantifying the numbers of those particles.

Previous studies have reported delayed appearance of the RE peak response relative to ApoB-48 and TAG (Cohn et al. 1989; Krasinski et al. 1990; Lovegrove et al. 1999). However, the delayed appearance of RE compared with the increase of plasma TAG is not a consistent finding in the

\footnotetext{
Abbreviations: Apo, apolipoprotein; AUC, area under the curve; CM, chylomicrons; RE, retinyl esters; RP, retinyl palmitate; TAG, triacylglycerol.

* Corresponding author: Dr Julie A. Lovegrove, fax +44 118931 0080, email J.A.Lovegrove@afnovell.reading.ac.uk
} 
literature. Several researchers who had quantified both plasma RE and TAG or RE, TAG and ApoB-48 found similar peak times (for review, see Lovegrove et al. 1999). The form of vitamin A used to label CM has been considered as a major cause for this apparent discrepancy (Krasinski et al. 1990; Lovegrove et al. 1999). Karpe et al. (1995) suggested that a delayed absorption of vitamin A in oily preparations cause this delayed appearance of RE in the plasma. The preparation of the RE used in the postprandial studies varies between studies, with some investigators using oil-soluble and others water-miscible preparations (for review, see Lovegrove et al. 1999). The RE in the oily preparations must be hydrolysed to retinol and solubilised by the bile salts before uptake by the enterocytes (Blomhoff et al. 1991), whereas vitamin A in aqueous preparations (present in the form of retinol) can be taken up directly.

The aim of the present study was to compare the use of commercially available 'water-miscible' and oil-soluble RP as markers of dietary-derived lipoproteins in healthy subjects, along with the simultaneous measurements of postprandial plasma TAG and ApoB-48 responses.

\section{Materials and methods}

\section{Subjects}

Nine healthy female volunteers (aged 31 (SD 11) years, BMI $22.7(\mathrm{SD} 3 \cdot 2) \mathrm{kg} / \mathrm{m}^{2}$ ) participated in the study. They were without history or clinical evidence of endocrine or liver disease, not taking medication that may influence blood lipoproteins, not undertaking more than $3 \times 20 \mathrm{~min}$ aerobic sessions a week. Their alcohol consumption was $<240 \mathrm{~g}$ alcohol/week. All subjects had fasting plasma measurements within the normal range. This study was approved by the Ethics Committee of the University of Reading, UK, and all subjects gave their written, informed consent to participate in the study.

\section{Postprandial study protocol}

Participants reported to the Human Investigation Unit at 08.30 hours, for postprandial investigations on two occasions separated by a 4-week period. They fasted overnight for $12 \mathrm{~h}$ and refrained from alcohol intake and strenuous exercises during the previous day. A standardised low-fat evening meal was given to the subjects the night before each study day. On arrival at the Investigation Unit, subjects were subjected to a pregnancy test prior to participating in the study to confirm they were not pregnant, due to known teratogenic effects of high doses of vitamin A. A cannula was inserted into the forearm vein and two fasting blood samples were collected over a period of $30 \mathrm{~min}$. Each subject then received a test meal of $40 \mathrm{~g}$ Rice Krispies, $72 \mathrm{~g}$ white bread, $14 \mathrm{~g}$ 'Flora' margarine, $50 \mathrm{~g}$ cheddar cheese, $200 \mathrm{~g}$ whole milk and a milk shake containing $6 \mathrm{~g}$ double cream, $150 \mathrm{~g}$ whole milk and $5 \mathrm{~g}$ Nesquick $^{\mathrm{TM}}$ (Nestle Ltd, Vevey, Switzerland). The test meal contained $47 \mathrm{~g}$ fat, $32 \mathrm{~g}$ protein and $91 \mathrm{~g}$ carbohydrate. The subjects consumed the test meal within $20 \mathrm{~min}$. Following consumption of the test meal, subjects were allowed free access to water or decaffeinated beverages without milk or sugar but no other food was allowed to be consumed until the end of the study.

\section{Retinyl palmitate labelling}

A dose of retinyl palmitate (RP) equivalent to $112 \mathrm{mg}$ in water-miscible or oil-soluble form (F. Hoffmann-La Roche Ltd, Basle, Switzerland) was given to each subject in random order, on two separate occasions, mixing the RP into the milk shake component of the test meal. All subjects tolerated the test meal and the RP dose. The start of the test meal was taken as the 0 time point and $15 \mathrm{ml}$ blood samples were taken, via the cannula, at $-30,0,30,60,90,120,150$, 180, 210, 240, 300, 360, 420 and 480 min time points.

\section{Analytical procedures}

Blood samples were collected into EDTA tubes and then plasma was separated by immediate centrifugation at $3000 \mathrm{rpm}$ for $15 \mathrm{~min}$. The plasma obtained was divided into portions into flat bottom tubes and stored at $-20^{\circ} \mathrm{C}$ for later analysis of plasma TAG, ApoB-48, RE, glucose, nonesterified fatty acids and total cholesterol. All samples for RP determination were protected from light. Samples for ApoB-48 determination had $50 \mathrm{ml} / 1$ added as preservative (Edelstein \& Scanu, 1986). The plasma concentrations of TAG, glucose, total cholesterol (using test kits supplied by Instrumentation Laboratories UK Ltd, Warrington, Ches., UK) and non-esterified fatty acids (using test kits Wako NEFC C kit; Alpha Laboratories Ltd, Eastleigh, Hants., UK) were determined on the Monarch automated analyser (Instrumentation Laboratories UK Ltd). RE were estimated by normal phase HPLC procedure (Ruotolo et al. 1992). The intra- and inter-assay CV were $<5 \%$ for all assays mentioned earlier. ApoB-48 was quantitated by a competitive enzyme-linked immunosorbant assay (Lovegrove et al. 1996). The intra-assay CV for the ApoB-48 analysis was $<5 \%$ and inter-assay CV were 14.8 and $11.4 \%$ at 0.94 and $3.94 \mathrm{mg}$ ApoB-48.

\section{Data and statistical analysis}

All analyses were performed using Statistical Analysis Systems statistical package, version 6.12 (SAS Institute Inc., Cary, NC, USA). Area under the postprandial response curve (AUC) and incremental AUC were calculated for TAG, RE and ApoB-48 (Matthews et al. 1990). Mean peak concentration (mean of the individual peak (highest) concentrations) and the mean peak time (mean time of peak concentration) were calculated for each individual. Repeated-measures analysis was performed on postprandial responses to test the effects of between subject factor (type of RP) and within-subject factor (time). The estimates of the postprandial concentrations at each time point for two types of RP were computed and the differences were tested for the significance. Differences between summary measurements for two types of RP were identified by ANOVA, followed by paired comparisons between two types of RP. Paired comparisons were also made on peak times of the markers of CM. 


\section{Results}

Postprandial plasma retinyl esters

The postprandial RE response to the test meals containing two types of RP significantly changed with time (repeatedmeasures analysis, $P<0 \cdot 0001$ ) and the type of RP showed a significant effect on the overall RE responses (RP main effect, $P<0 \cdot 002$ ). The patterns of the RE response to the test meals with different types of RP were also significantly different (RP $\times$ time interaction, $P<0 \cdot 0001$ ). Following the consumption of the test meal with water-miscible RP, the plasma RE increased sharply and reached a peak concentration between 90 and $210 \mathrm{~min}$ (Fig. 1(a)) and then showed a rapid decrease towards fasting levels. In contrast,

(a)

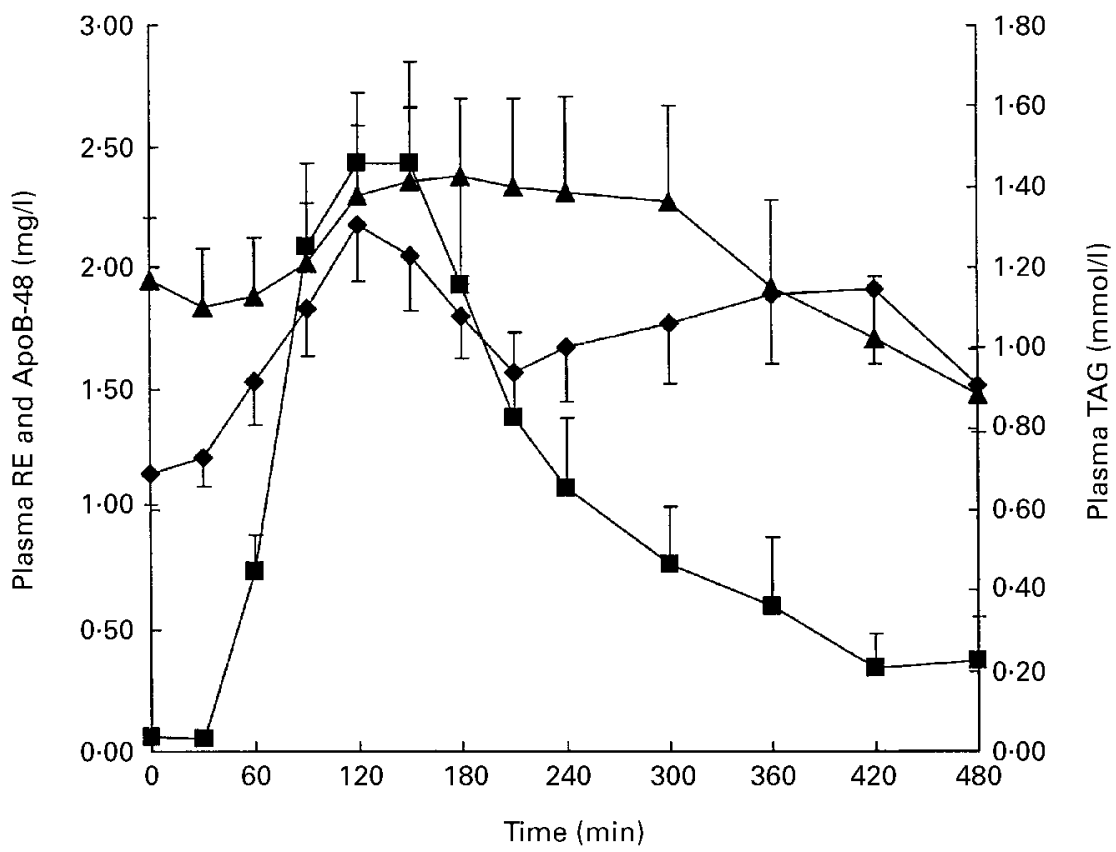

(b)

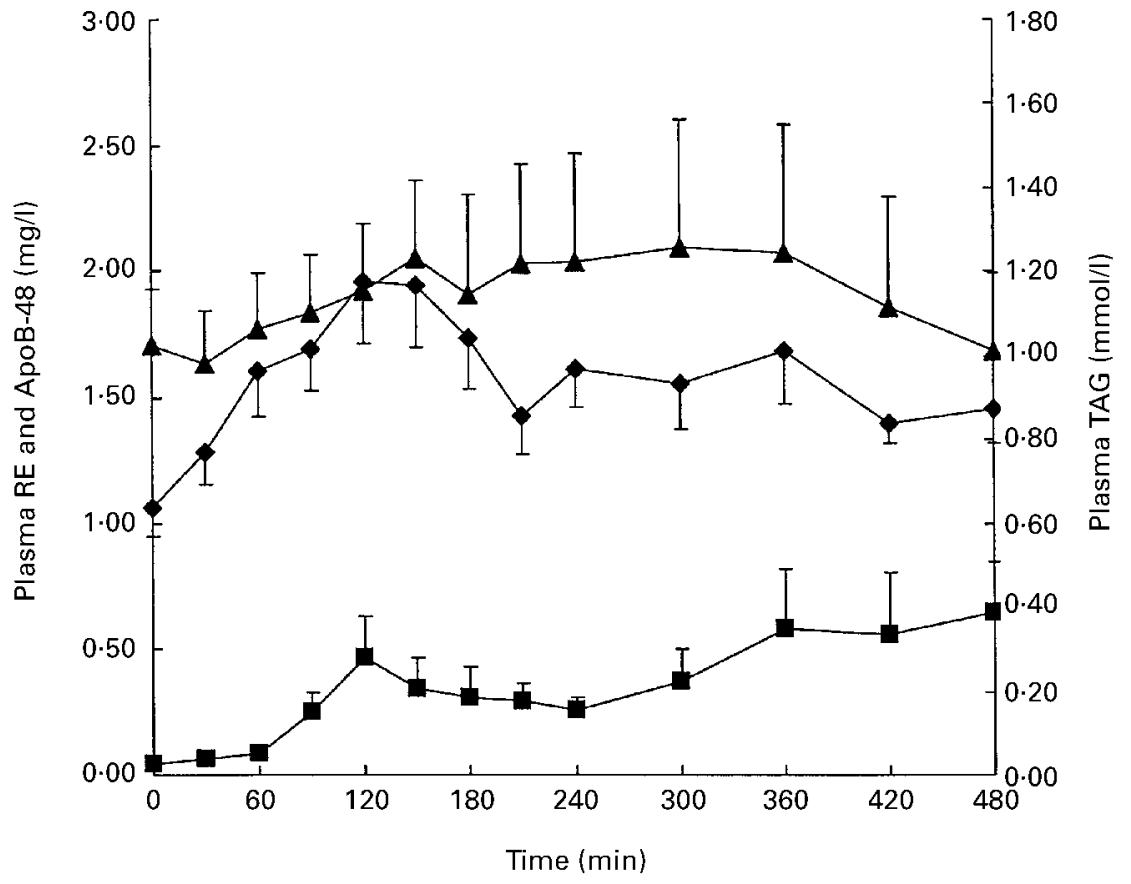

Fig. 1. Postprandial apolipoprotein (Apo) B-48 $(\bullet)$, triacylglycerols (TAG, $\mathbf{\Delta}$ ) and retinyl esters $(R E, \quad$ ) responses to the test meals containing (a) water-miscible and (b) oil-soluble RP in nine female subjects. For details of subjects and procedures, see p. 428. Values are means with their standard errors represented by vertical bars. 
plasma RE response to the oil-soluble RP containing test meal showed a much less marked increase in concentration following the test meal and reached a first smaller peak at about $90-120 \mathrm{~min}$ and a further increase in concentrations after $300 \mathrm{~min}$ that was sustained until the end of the $8 \mathrm{~h}$ study period (Fig. 1(b)). The estimates of the plasma RE concentrations after the water-miscible RP were significantly higher $(P<0 \cdot 01)$ between 60 to $240 \mathrm{~min}$ compared with the response to the test meal with oil-soluble RP. The $\mathrm{RE}$ response to the test meal containing water-miscible RP showed lower concentrations than that of test meal containing oil-soluble RP after $360 \mathrm{~min}$.

The AUC $(P<0 \cdot 001)$ and mean peak concentration $(P<0 \cdot 01)$ for the RE response were significantly higher after water-miscible RP compared with oil-soluble RP (Table 1). Although oil-soluble RP showed an earlier small peak around 90-120 min, the highest concentration (peak concentration) was observed at a significantly $(P<0 \cdot 001)$ later time point (327 (SE 54) min) than that of watermiscible RP (117 (SE 12) min).

\section{Postprandial plasma triacylglycerols}

Repeated-measures analysis showed significant changes of TAG with time $(P<0 \cdot 0001)$ with no main effect of the type of RP on the postprandial TAG response. However, there were significantly different patterns of TAG response over the $8 \mathrm{~h}$ study period $(P<0.01)$. The plasma TAG concentrations following the test meal containing oilsoluble RP tended to be lower than that following the test meal containing water-miscible RP up to $360 \mathrm{~min}$. Thereafter, plasma TAG response to the test meal containing oilsoluble RP was higher than that following the test meal containing water-miscible RP. There was no significant difference in the AUC, incremental AUC, peak concentration and peak time of the postprandial plasma TAG when consumed different types of RP (Table 1).

\section{Postprandial plasma apolipoprotein B-48, non-esterified fatty acids, glucose and total cholesterol}

There were no significant differences in the magnitude and patterns of the postprandial responses of the measurements mentioned earlier. The AUC, incremental AUC, peak concentration and peak time were not significantly different between the two types of RP.

\section{Peak times of retinyl esters, apolipoprotein B-48 and triacylglycerol concentration}

The RE reached the maximum concentration significantly earlier than that of TAG peak concentration when the watermiscible RP was consumed with the test meal (Table 1). In contrast, after consumption of the oil-soluble RP with the same test meal, RE reached the mean peak concentration at 327 (SE 54) min (prominent peak), which was later than that of ApoB-48 and TAG. There were no significant differences in peak times of the postprandial plasma concentrations of ApoB-48 and TAG for both types of RP.

\section{Discussion}

The results of the present study showed that large doses of water-miscible and oil-soluble RP administered with a fat load in order to label dietary-derived lipids result in different postprandial responses of plasma RE. Similar

Table 1. Postprandial measurements of retinyl ester, triacylglycerol and apolipoprotein B-48 for test meals containing water-miscible and oil-soluble retinyl palmitate§

(Mean values with their standard errors for nine female subjects)

\begin{tabular}{|c|c|c|c|c|}
\hline & \multicolumn{2}{|c|}{ Water-miscible RP } & \multicolumn{2}{|c|}{ Oil-soluble RP } \\
\hline & Mean & SE & Mean & SE \\
\hline \multicolumn{5}{|l|}{ RE } \\
\hline AUC (mg/min per I) & $491^{\star \star \star}$ & 86 & 177 & 43 \\
\hline Peak concentration (mg/l) & $2 \cdot 79^{\star \star}$ & 0.37 & 1.00 & 0.24 \\
\hline Peak time $(\min ) \|$ & $117^{* * *}+\dagger$ & 12 & $327 \dagger \ddagger$ & 54 \\
\hline \multicolumn{5}{|l|}{ TAG } \\
\hline AUC $(\mathrm{mmol} / \mathrm{min}$ per I) & 590 & 82 & 556 & 104 \\
\hline Incremental AUC (mmol/min per I) & 27 & 46 & 64 & 55 \\
\hline Peak concentration $(\mathrm{mmol} / \mathrm{l})$ & 1.57 & 0.24 & 1.43 & 0.29 \\
\hline Peak time & 182 & 18 & 182 & 30 \\
\hline \multicolumn{5}{|l|}{ ApoB-48 } \\
\hline AUC (mg/min per I) & 828 & 92 & 759 & 65 \\
\hline Incremental AUC (mg/min per I) & 278 & 67 & 251 & 57 \\
\hline Peak concentration $(\mathrm{mg} / \mathrm{l})$ & 2.48 & 0.27 & $2 \cdot 16$ & 0.26 \\
\hline Peak time (min) & 152 & 12 & 140 & 10 \\
\hline
\end{tabular}

$\mathrm{RP}$, retinyl palmitate; RE, retinyl ester; AUC, area under the curve; TAG, triacylglycerol; Apo, apolipoprotein.

Mean values were significantly different from those of the oil-soluble RP group (paired $t$ test): ${ }^{* *} P<0.01,{ }^{* * *} P<0.001$.

Mean values were significantly different from those of TAG in the same treatment group (paired $t$ test) † $P<0.03$, t十 $P<0.002$.

Mean value was significantly different from that of ApoB-48 in the same treatment group (paired $t$ test): $\ddagger P<0.06$.

$\S$ For details of subjects and procedures, see p. 428

II Time to reach the most prominent peak. 
ApoB-48 responses and greater rise in plasma $\mathrm{RE}$ concentrations in the first $1-4 \mathrm{~h}$ after the test meal with water-miscible RP suggest that the ApoB-48 containing lipoproteins carried larger amounts of RE in plasma during that period than when oil-soluble RP was consumed. These findings indicate faster and greater absorption of watermiscible RP and/or faster packaging of them into CM. It has been shown that high doses of vitamin A administered in an aqueous preparation results in greater postabsorptive plasma vitamin A levels than in an oily preparation in children (Lewis et al. 1947). Johnson et al. (1992) demonstrated that physiologic doses of 'water-soluble' vitamin A are absorbed very effectively compared with oil-soluble preparations in patients with pancreatic disease. RP in the oily preparations must be hydrolysed to retinol and solubilised by bile salts before being taken up by enterocytes (Ong, 1994), whereas the vitamin $\mathrm{A}$ in the form of water-soluble retinol, which has been used in many postprandial investigations to label dietary-derived fats, could be taken up by the enterocytes directly. However, the exact mechanism of uptake by enterocytes of water-miscible types of RP used in the present study is not known. Because the plasma RE showed higher concentrations following the RP in water-miscible preparation, a faster rate of hydrolysis by pancreatic RE hydrolase and/or rapid absorption by enterocytes can be suggested. Alternatively, it can be hypothesised that formation of micelles may not be required for absorption of the aqueous preparations because RP in this preparation is already dissolved in a polar matrix.

When the subjects had oil-soluble RP in the present study, a slower rise in the plasma RE concentrations until $4 \mathrm{~h}$ after the test meal was observed and thereafter, concentrations continued to increase. The delayed and/or lower absorption, delayed re-esterification and incorporation of RE into CM can be suggested as the possible reasons for the delayed rise in plasma RE concentrations when oil-soluble RP was consumed. The delayed $\mathrm{RE}$ response relative to TAG following the ingestion of oil-soluble RP can also be explained by differences in the metabolism of these substances during CM synthesis and catabolism. It is believed that RE given with a fat load are hydrolysed over a similar rate and time course as the hydrolysis of dietary cholesteryl esters in the gut rather than dietary TAG, and the rates of incorporation into the $\mathrm{CM}$ also differ from that of TAG (Ong, 1994). However, the pattern of the RE response following water-miscible RP in the present study indicated better concordance with plasma TAG response indicating a similar rate of packaging of RE relative to TAG into CM when water-miscible RP was used. However, different patterns of plasma RE and ApoB-48 responses suggests that vitamin A labelling of $\mathrm{CM}$ seems not to be a valid approach for quantifying the number of TAG-rich lipoprotein particles of intestinal origin, at least during the late postprandial period.

More frequent blood sampling, high sensitivity of the HPLC equipment used and smaller numbers of subjects with considerable variation in their RE responses can be suggested as the reasons for observing a small earlier peak in the present study. Although the overall degree of postprandial plasma TAG response was not affected by the type of RP, the changing patterns showed some differences with higher concentrations of plasma TAG up to $6 \mathrm{~h}$ when water-miscible RP was consumed and higher plasma concentrations from $6-8 \mathrm{~h}$ when oil-soluble RP was consumed. The most likely explanation is that dietary TAG and RE will have competed for the formation of micelles and the action of pancreatic lipases in the duodenum, with this effect most marked in the early postprandial period when the dietary load of TAG was at its highest. As a result of the different timing and/or less competition of water-miscible RE with TAG for the formation of micelles and the action of lipases, the latter can be absorbed more rapidly and appear in the plasma at higher concentrations during the early postprandial period than when oil-soluble RE was ingested.

In conclusion, the results suggest more rapid absorption and higher and earlier peak responses of plasma RE when water-miscible RP is consumed, in contrast to the delayed initial appearance and later sustained higher concentrations of plasma RE during late postprandial period when oilsoluble RP is consumed. The RE response to the watermiscible RP showed better concordance with plasma TAG and ApoB-48 responses than that of oil-soluble RP.

\section{Acknowledgements}

Authors wish to thank Drs John Wright and Kim Jackson for their help in postprandial studies. KDRRS is sponsored by the Commonwealth Scholarship Commission, UK.

\section{References}

Blomhoff R, Green MH, Green JB, Berg T \& Norum KR (1991) Vitamin A metabolism: New perspectives on absorption, transport, and storage. Physiological Reviews 71, 951-990.

Cohn JS, McNamara JR, Krasinski SD, Russell RM \& Schaefer EJ (1989) Role of triglyceride-rich lipoproteins from the liver and intestine in the etiology of postprandial peaks in plasma triglyceride concentration. Metabolism 38, 484-490.

Cortner JA, Coates PM, Le N-A, Cryer DR, Ragni MC, Faulkner A \& Langer T (1987) Kinetics of chylomicron remnant clearance in normal and hyperlipoproteinemic subjects. Journal of Lipid Research 28, 195-206.

Edelstein C \& Scanu AM (1986) Precautionary measures for collecting blood destined for lipoprotein isolation. Methods in Enzymology 128, 151-155.

Goodman DS, Bolmstrand B, Werner B, Huang HS \& Shiratori T (1966) The intestinal absorption and metabolism of vitamin A and beta-carotene in man. Journal of Clinical Investigation 45, $1615-1623$.

Goodman DS, Huang HS \& Shiratori T (1965) Tissue distribution and metabolism of newly absorbed vitamin A in the rat. Journal of Lipid Research 6, 390-396.

Hazzard WR \& Bierman EL (1976) Delayed clearance of chylomicron remnants following vitamin A-containing oral fat loads in broad beta disease (type III hyperlipoproteinaemia). Metabolism 25, 771-801.

Johnson EJ, Krasinski SD, Howard LJ, Alger SA, Dutta SK \& Russell RM (1992) Evaluation of vitamin A absorption by using oil-soluble and water-miscible vitamin A preparations in normal adults and in patients with gastrointestinal disease. American Journal of Clinical Nutrition 55, 857-864.

Karpe F, Bell M, Björkegren J \& Hamsten A (1995) Quantification of postprandial triglyceride-rich lipoproteins in healthy men by retinyl ester labelling and simultaneous measurement of 
apolipoprotein B-48 and B-100. Arteriosclerosis, Thrombosis and Vascular Biology 15, 199-207.

Krasinski SD, Cohn JS, Russell RM \& Schaefer EJ (1990) Postprandial plasma vitamin A metabolism in humans: A reassessment of the use of plasma retinyl esters as markers for intestinally derived chylomicrons and their remnants. Metabolism 39, 357-365.

Lewis JM, Bodansky O, Birmingham J \& Cohlan SQ (1947) Comparative absorption, excretion and storage of oily and aqueous preparation of vitamin A. Journal of Pediatrics 31, 496-508.

Lovegrove JA, Isherwood SG, Jackson KG, Williams CM \& Gould BJ (1996) Quantitation of apolipoprotein B-48 in triacylglycerol-rich lipoproteins by a specific enzyme-linked immunosorbant assay. Biochimica et Biophysica Acta 1301, 221-229.

Lovegrove JA, Jackson KG, Murphy MC, Brookes CN, Zampelas A, Knapper JME, Wright JW, Gould BJ \& Williams CM (1999) Markers of intestinally-derived lipoproteins: application to studies of altered diet and meal fatty acid composition. Nutrition, Metabolism and Cardiovascular Diseases 9, 9-18.
Matthews JNS, Altman DG, Campbell MJ \& Royston P (1990) Analysis of serial measurements in medical research. British Medical Journal 300, 230-235.

Ong DE (1994) Cellular transport and metabolism of vitamin A: Roles of cellular retinoid-binding proteins. Nutrition Reviews 52, S24-S31.

Ruotolo G, Zhang H, Bentsianov V \& Le NA (1992) Protocol for the study of the metabolism of retinyl esters in plasma lipoproteins during postprandial lipaemia. Journal of Lipid Research 33, 1541-1549.

Thompson KH, Hughes LB \& Zilversmit DB (1983) Lack of secretion of retinyl esters by livers of normal and cholesterol-fed rabbits. Journal of Nutrition 113, 1995-2001.

Wilson DE, Chan IF \& Ball M (1983) Plasma lipoprotein retinoids after vitamin A feeding in normal man: Minimal appearance of retinyl esters among low density lipoproteins. Metabolism 32, 514-517.

Zilversmit DB (1979) Atherogenesis: a postprandial phenomenon. Circulation 60, 473-485. 